\title{
Perspective
}

\section{What does our genome encode?}

\author{
John A. Stamatoyannopoulos ${ }^{1}$ \\ Departments of Genome Sciences and Medicine, University of Washington School of Medicine, Seattle, Washington 98195, USA
}

\begin{abstract}
In its first production phase, The ENCODE Project Consortium (ENCODE) has generated thousands of genome-scale data sets, resulting in a genomic "parts list" that encompasses transcripts, sites of transcription factor binding, and other functional features that now number in the millions of distinct elements. These data are reshaping many long-held beliefs concerning the information content of the human and other complex genomes, including the very definition of the gene. Here I discuss and place in context many of the leading findings of ENCODE, as well as trends that are shaping the generation and interpretation of ENCODE data. Finally, I consider prospects for the future, including maximizing the accuracy, completeness, and utility of ENCODE data for the community.
\end{abstract}

Almost exactly 10 years ago, a "Workshop on the Comprehensive Extraction of Biological Information from Genomic Sequence" endorsed the formation of a public consortium to undertake comprehensive annotation of all functional elements encoded in the human genome, a logical encore to the production of the genome sequence itself. At that time, in spite of general optimism for the cause, few things were clear. The task lacked precedent, obscuring its true scope; the requisite technologies were either nascent or, at the time of the workshop, not yet imagined; and, on the heels of the mouse genome sequence, the very role of experimental approaches was challenged by the burgeoning promise of comparative genomics. What was not in doubt was the commitment of NHGRI to build on the success of the Human Genome Project, which had yet to announce its finished sequence.

In this environment, The ENCODE Project Consortium (ENCODE) took form. A four-year pilot phase initiated in 2003 focused on a carefully selected $1 \%$ of the human genome and was oriented chiefly toward the deployment at scale and assessment of experimental and computational genomic technologies for localization of functional elements. In 2007, the pilot project was succeeded by the scale-up to a production phase that saw the expansion of ENCODE annotation efforts to the entire genomic sequence. In a happy and fateful coincidence, the ENCODE scale-up commenced contemporaneously with the introduction of massively parallel "next-generation" sequencing technologies-a development that was rapidly exploited by ENCODE groups to replace wholesale the assays that had been utilized during the pilot phase. Indeed, ENCODE groups played leading roles in the genesis and deployment of many staple genomic assays in wide use today, including the first ChIP-plus-sequencing assays (ChIP-seq) for transcription factors (Johnson et al. 2007; Robertson et al. 2007) and histone modifications (Barski et al. 2007; Mikkelsen et al. 2007), as well as pioneering RNA sequencing assays (RNA-seq) (Mortazavi et al. 2008), among others.

The ENCODE production phase has produced thousands of genome-wide data sets during the past five years, yielding deep insights into genome function, and ENCODE is now poised for a further multi-year expansion phase. As such, the present juncture provides a useful vantage from which to reflect on the ENCODE's accomplishments, challenges, and prospects. Here, I first discuss how ENCODE has influenced our conception of genome structure

\footnotetext{
${ }^{1}$ Corresponding author

E-mail jstam@u.washington.edu

Article is at http://www.genome.org/cgi/doi/10.1101/gr.146506.112.

Freely available online through the Genome Research Open Access option.
}

and content, and the utility of function-driven versus purely sequence-based approaches to genome annotation. Second, I consider major trends that are shaping both the nature of ENCODE data and how those data are conceptualized and used. Finally, I discuss key challenges confronting the next phase of the ENCODE endeavor.

\section{Reading the living genome}

\section{Functional elements, then and now}

Although the ENCODE project formally originated in the postgenome era, its intellectual origins lie some 40 years earlier with the concept that genomes contain discrete, linearly ordered units that can be connected with specific functional features or processes (Jacob and Monod 1961). A cornerstone of ENCODE has been the use of biochemical signatures to identify functional elements specified by the genomic sequence. In part, this represents a departure from the widely accepted reductionist approach to genome function, in which iterative dissection by truncation or editing of larger sequences that encompass a given functional activity was coupled to an experimental read-out of that activity. The reductionist approach provided a powerful experimental paradigm and was widely applied to define and understand the signals that direct transcription initiation, splicing, and other basic processes, and to expose the transcription factor binding elements that comprise the sequence "atoms" of gene regulation.

The biochemical signature strategy, which developed in parallel with reductionism, was motivated by the recognition of common biochemical or biophysical events that invariably attended certain types of noncoding functional elements. This strategy found its first expression in the discovery that active promoters were marked by alterations in chromatin structure that gave rise to nuclease hypersensitivity of the underlying DNA (Wu et al. 1979; Wu 1980). This signature was subsequently sought over entire genomic loci (Stalder et al. 1980) and resulted in definition of the first cellular enhancers (Banerji et al. 1983) and other types of transcriptional control elements (Forrester et al. 1986; Grosveld et al. 1987; Chung et al. 1993). Reductionism was, in turn, applied to the biochemically defined elements, revealing them to be densely populated by recognition sequences for DNA-binding proteins (Emerson et al. 1985; Strauss and Orkin 1992), motivating, in turn, the development of site-specific factor occupancy assays such as ChIP (Gilmour and Lis 1984; Solomon and Varshavsky 1985). Subsequently, the recognition that histone modification patterns could suggest transcription factor occupancy patterns (Lee et al. 1993) and functional characteristics of adjacent regula- 
tory regions (Bernstein et al. 2002, 2005) led to the identification of biochemical signatures that could be exploited on a genomic scale across multiple cell types (The ENCODE Project Consortium 2007; Heintzman et al. 2007; Mikkelsen et al. 2007). In a similar vein, RNA transcripts were increasingly used to annotate both sites of transcript origination (both coding and noncoding) as well as the nuances of processed transcript structure. Eventually, the accumulation of large amounts of data connecting biochemical signatures of specific DNA regions with particular functional activities set the stage for the generic large-scale mapping of functional elements. Critically, this could now be undertaken without detailed knowledge of downstream functions. For example, genes could be annotated without knowledge of the function of their protein products, and regulatory DNA regions could be annotated without knowledge of their ultimate functional consequences for a given gene-or even what their target gene might be.

At the outset of The ENCODE Pilot Project in 2003, the number of transcriptional regulatory elements defined using traditional approaches, including the pre-genome application of biochemical signatures such as DNase I hypersensitivity, stood at perhaps a few hundred. At the conclusion of the first ENCODE production phase, this total has increased nearly 10,000-fold. However, the number of such elements for which we possess classical experimental validation is still in the low hundreds. Nonetheless, the information that can be extracted from this vast cache of elements is breathtaking. By studying the trans-cellular patterning of biochemical signatures, we gain telling insights into elements responsible for cell-selective regulation of transcript expression (Arvey et al. 2012; Djebali et al. 2012a; Thurman et al. 2012), the combinatorial patterns of transcription factors (TFs) that occupy them (Gerstein et al. 2012; J Wang et al. 2012b), and their likely genic targets (Sanyal et al. 2012; Thurman et al. 2012). Although ENCODE was conceived as a genome annotation project fundamentally focused on the linear organization of sequence elements, it is now becoming clear that connectivity between linear elements is an intrinsic part of this annotation-from splicing, to long-range chromatin interactions (de Wit and de Laat 2012), to transcription factor networks (Gerstein et al. 2012; Neph et al. 2012a). How these insights will be systematically integrated into ENCODE annotations remains a significant challenge. And just how much functional validation using traditional approaches will ultimately be required is unclear-a topic I consider further below.

\section{The genus 'gene'}

The dual concept of the gene both as the agent of heredity and as a physical, information-laden entity embodied in a specific DNA sequence has dominated modern biology. Great emphasis has been placed on the accurate and comprehensive annotation of genes in the human genome and across the spectrum of sequenced organisms. Over the last 10 years, ENCODE data have engendered numerous fundamental observations concerning the organization of transcription that have collectively provided deep insights into genome function as well as continually reshaped our conception of a gene. These include the recognition of pervasive transcription (Cheng et al. 2005; Manak et al. 2006; The ENCODE Project Consortium 2007; Kapranov et al. 2007; Efroni et al. 2008; Clark et al. 2011), long-range splicing and chimeric transcripts (Djebali et al. 2012b; Frenkel-Morgenstern et al. 2012), promoter-associated small RNAs (Affymetrix/Cold Spring Harbor Laboratory ENCODE Transcriptome Project 2009), and the splicing-chromatin connection (Tilgner et al. 2009), among other findings.
Although the gene has conventionally been viewed as the fundamental unit of genomic organization, on the basis of ENCODE data it is now compellingly argued that this unit is not the gene but rather the transcript (Washietl et al. 2007; Djebali et al. 2012a). On this view, genes represent a higher-order framework around which individual transcripts coalesce, creating a polyfunctional entity that assumes different forms under different cellular states, guided by differential utilization of regulatory DNA. This concept is implicit in the organization of the GENCODE effort to annotate transcriptional units-protein-coding and noncoding, alive and dead (pseudogenes)—by means of the careful compilation, analysis, and validation of RNA transcripts from diverse sources (Derrien et al. 2012; Djebali et al. 2012a; Harrow et al. 2012; Howald et al. 2012). This effort has resulted in a new standard reference annotation covering everything from alternative transcriptional start sites to antisense transcripts, and it has anchored and empowered numerous integrative analyses. Indeed, the GENCODE annotation is used in some form by every ENCODE paper in this issue, and those contemporaneously published in other journals.

Intensive probing of the RNA compartment has further deepened our appreciation of the extreme diversity and complexity of transcriptional processes and the very nature of pervasive transcription. Sequencing of RNAs from nuclear subcompartments such as the nucleolus or chromatin has revealed that even seemingly simple gene structures may be hiding an astonishing variety of transcript forms (Djebali et al. 2012a). Moreover, the systematic analysis of nuclear transcripts now clearly supports cotranscriptional splicing as a frequent mechanism of transcript processing (Tilgner et al. 2012). These studies and other recent reports detailing deep probing of the RNA world (Mercer et al. 2011) affirm the centrality of the transcript in genomic organization, while highlighting both the opportunity and the daunting challenge of comprehensive transcriptome annotation.

\section{The chromatin-transcription continuum}

The interplay between transcription and chromatin has been a topic of intense study for over 30 years, during which time our view of the role of chromatin in modulating transcription has evolved dramatically, from a static physical obstacle that must be negotiated during transcription to a complex entity that dynamically exchanges information with a transcribing polymerase to facilitate its transit across the genic landscape. The past five years in particular have witnessed a striking convergence of our views connecting chromatin and transcription, spurred by observations grounded in ENCODE data.

Transcription originating from enhancer elements was first described over 20 years ago (Tuan et al. 1992) and has recently reemerged through analysis of deep RNA-seq data (Kim et al. 2012). This phenomenon has now been extensively documented by ENCODE (Djebali et al. 2012a) and is detected predominantly at distal DNase I hypersensitive sites that are flanked by H3K4me1, H3K27ac, and H3K9ac histone modifications. Unlike promoters, the enhancer-originated transcripts derive overwhelmingly from nuclear nonpolyadenylated RNA and are thus missing the large fraction of polyadenylated transcripts characteristic of canonical promoters. The rate of enhancer-originated transcription varies widely, and generally occurs at a substantially lower level than promoters.

The association of trimethylation at histone $\mathrm{H} 3$ lysine 4 (H3K4me3) with transcription initiation at human genes is well described (Wang et al. 2008; Ernst et al. 2011) and is a direct outgrowth of studies in yeast (Bernstein et al. 2002; Santos-Rosa et al. 2002). More striking and unexpected was the discovery that 
patterns of histone modification (H3K36me3) and nucleosome location preference within gene bodies reflect organizational features of mature transcript structure such as exons, as well as their splicing frequency (Kolasinska-Zwierz et al. 2009; Tilgner et al. 2009). However, both the mechanism(s) giving rise to these phenomena and their implications for global genome function remain largely obscure. It is likely, however, that many more subtle connections between chromatin modification and transcript structure lie waiting to be uncovered in ENCODE data, some of which may be brought to light with the increasing cost effectiveness of deeper sequencing that will, in turn, enable finer parsing of the chromatin landscape.

How the nuclear machinery executes a high-precision operation such as splicing over genomic distances that may exceed $1 \mathrm{Mb}$ is currently unknown. The most straightforward explanation is that, analogous to enhancers and their target promoters, these transcript components are physically approximated to one another through direct chromatin interactions. Such hypotheses are now directly testable by cross-analysis of long-range splicing data with ENCODE 5C (Sanyal et al. 2012) and ChIA-PET results (Li et al. 2012).

Viewed collectively, ENCODE data are increasingly pointing to the conclusion that chromatin and transcription are not discrete genomic forces that collide in the context of gene expression. Rather, they represent a continuum of activities, from the infrequent generation of transcripts at distal regulatory DNA, to regions of high transcriptional output that are marked by pervasive alterations in chromatin state. It is certain that many additional features of the transcription-chromatin connection remain to be uncovered within extant ENCODE data. Going forward, deeper probing of both the RNA and chromatin compartments through advancing sequencing throughput will perhaps bring these features to light more quickly.

\section{Regulatory DNA: More than meets the eye}

It is still widely believed that functional elements, from exons to regulatory DNA, are relatively rare features of the genomic landscape. In the case of regulatory DNA, this is certainly true within the context of an individual cell type, where DNase I hypersensitive sites and associated transcription factor occupancy sites mapped by ChIP-seq encompass on the order of $1 \%-2 \%$ of the genome-a compartment roughly the size of the exome. However, because the majority of regulatory DNA regions are highly cell type-selective (The ENCODE Project Consortium 2012; Thurman et al. 2012), the genomic landscape rapidly becomes crowded with regulatory DNA as the number of cell types and states assayed increases. Even after assaying more than 120 distinct cell types, this trend shows little evidence of saturation (The ENCODE Project Consortium 2012). It is thus not unreasonable to expect that $40 \%$ and perhaps more of the genome sequence encodes regulatory information-a number that would have been considered heretical at the outset of the ENCODE project. It is important to recognize, however, that this figure encompasses regulatory regions wherein only a subset of the individual nucleotides are under strong evolutionary constraint, such as those at critical contact positions for transcription factor recognition (Neph et al. 2012b).

It is also widely assumed that roughly half of the human genome sequence has been laid waste by transposable elements and other classes of repetitive sequences, which have repeatedly and haphazardly pummeled the genome at various evolutionary intervals. These regions were all but invisible during the ENCODE pilot phase, where they were intentionally masked from microarray designs. But this situation changed dramatically with se- quence tag-based assays: Even modest read lengths (36-50 bp) have the potential to align uniquely with over $85 \%$ of the genome sequence, and thus to annotate a majority of transposable elements. In marked contrast to the prevailing wisdom, ENCODE chromatin and transcription studies now suggest that a large number of transposable elements encode highly cell type-selective regulatory DNA that controls not only their own cell-selective transcription, but also those of neighboring genes (Djebali et al. 2012a; Thurman et al. 2012). Far from an evolutionary dustbin, transposable elements appear to be active and lively members of the genomic regulatory community, deserving of the same level of scrutiny applied to other genic or regulatory features.

\section{Leaving the flat genome behind}

Gene regulation is fundamentally a three-dimensional (3D) process involving dynamic interactions between genomic DNA and the nuclear protein machinery. And yet, common conceptions of regulatory genomic processes are typically unidimensional, playing out over linear genome distance versus physical nuclear distance. ENCODE has played a key role in shifting this paradigm by providing key insights into the topology of gene regulation at two levels-nuclear structure and organization, and physical connectivity among cis-regulatory elements.

From our present vantage, it may seem remarkable that the discovery of the connection between 3D nuclear chromatin architecture and mammalian gene regulation (Weintraub and Groudine 1976) predated many one-dimensional representations that have populated the literature for the past three decades. Enabled by serial innovations in the quantitative analysis of chromatin interactions by Dekker and colleagues (Dekker et al. 2002; Dostie et al. 2006; Lieberman-Aiden et al. 2009; Nora et al. 2012), the visionary conception of Weintraub and Groudine is systematically taking form within the context of ENCODE. HiC data (Lieberman-Aiden et al. 2009) now provide global contact maps of nuclear chromatin that are sufficiently detailed as to enable reconstruction of the folding pattern of chromosomes within the confines of the nucleus, and to define major chromatin compartments. A key challenge is resolution, which increases only slowly with multiplicative increases in sequencing depth. However, recent increases in sequencing throughput have enabled deeper sampling, shedding further light on large-scale chromatin interactions and chromosomal domain architectures (Dixon et al. 2012; Nora et al. 2012).

The discovery of long-range cis-regulatory elements such as the immunoglobulin enhancer (Banerji et al. 1983) and the betaglobin Locus Control Region (Forrester et al. 1986; Grosveld et al. 1987) immediately raised the question of how such distal regulatory regions communicate with their target gene(s) and, more broadly, how genes and regulatory DNA are "wired" along a chromosome. Specific physical interactions appear to be a general property of long-range regulatory control, and are directly assayable with 3C (Dekker et al. 2002) or, at many elements in parallel, by 5C (Dostie et al. 2006). Systematic application of 5C to assay all mutual chromatin interactions over The ENCODE Pilot Project regions comprising $1 \%$ of the genome has now enabled a comprehensive synthesis of interactions between promoters and distal elements including likely enhancers and CTCF-occupied sites (Sanyal et al. 2012). Composite interaction technologies developed within ENCODE such as ChIA-PET-essentially a combination of ChIP-seq with a chromosome conformation capture assay-are further illuminating the 3D connectivity of human 
genes with one another and with their respective controlling elements (Li et al. 2012).

Together, the chromosome conformation capture-based approaches have probed local, domain, and global level interactions across the mammalian genome (de Wit and de Laat 2012). Although great progress in mapping genome connectivity has been achieved in a few short years, significant challenges remain, both technical and conceptual. The resolution of chromatin interaction assays is generally limited by restriction fragments, and these assays have a "blind" spot around anchor regions or around highly interacting elements such as promoters, where nonspecific local interactions may obscure more specific connections.

One of the greatest technical challenges facing ENCODE is to transform its linear genomic signals into nuclear space, without sacrificing resolution. Such a transformation would dramatically close the gap between cis-regulatory architecture and nuclear architecture, bringing us full circle in the journey begun by the pioneering experiments of Weintraub and Groudine more than three decades ago.

\section{From elements to networks}

Transcription factors interact with one another at three basic levels: direct protein-protein interactions; cooperative interactions engendered by binding within the same cis-regulatory element; and cross-regulatory interactions resulting from the binding of one transcription factor within the regulatory DNA regions controlling another factor. Extended across all transcription factors active within a given cell type, the last of these creates a transcription factor regulatory network that functions as a coherent system to process complex biological signals and confer robustness (Neph et al. 2012a).

Transcription factor regulatory networks can now be mapped systematically using two types of ENCODE data-ChIP-seq for individual transcription factors (Gerstein et al. 2012) and genomic DNase I footprinting (Neph et al. 2012a,b). The resulting networks can be rendered either as a regulatory "cloud" or as a hierarchy of interacting factors (Gerstein et al. 2012). However, for most users of ENCODE data, the greatest interest will lie in specific subnetworks that comprise the wiring of small cohorts of transcription factors, such as those involved in pluripotency or hematopoietic differentiation (Neph et al. 2012a). Deepening of ENCODE annotations through the survey of increasing numbers of transcription factors by ChIP-seq, and increasing numbers of DNase I footprints by deeper sequencing and survey of additional cell types, will further enrich our understanding of the human transcription factor network and how it feeds back to the level of individual regulatory DNA regions-and ultimately to other chromatin features and transcript production.

\section{Decoding disease}

The ENCODE production phase was initiated at the height of excitement over genome-wide association studies, nearly a thousand of which have since been performed. At that time, the prospects for convergence between the two initiatives seemed limited at best. Five years on, building on initial observations (Gaulton et al. 2010; Ernst et al. 2011), it is now apparent that a significant proportion of strongly disease- or trait-associated variants emerging from genome-wide association studies (GWASs) localize within regulatory DNA marked by DNase I hypersensitive sites and selected TFs (The ENCODE Project Consortium 2012; Maurano et al. 2012a; Schaub et al. 2012). Beyond simple enrichment, analysis of an expanded range of cell and tissue types reveals systematic and deep connections between the tissue and developmental stage selectivity with which disease- and trait-associated variants localize within regulatory DNA; the transcription factor recognition sequences perturbed by these variants; and the networks formed by these transcription factors (Maurano et al. 2012a). The ability to connect distal DHSs systematically with their cognate genes (Thurman et al. 2012) has now revealed many links between variants in regulatory DNA and distant genes that plausibly explain the disease associations (Maurano et al. 2012a).

It is also clear that the modest (but highly significant) overall degree of enrichment of disease- and trait-associated variants within regulatory DNA is an inappropriate measure that incorporates both noise within the GWAS data and the heterogeneous mix of cell types examined, many of which are peripheral to certain traits. In contrast, striking cell-selective enrichment of GWAS variants may be observed in pathogenic cell types, for example, the enrichment of variants associated with Crohn's disease in DHSs from Th1 T cells (The ENCODE Project Consortium 2012) or, even more prominently, in Th17 T cells, which play a leading role in Crohn's pathogenesis (Maurano et al. 2012a). Significantly, strong cell-selective enrichments are observed for hundreds of variants that fall below the canonical genome-wide significance threshold $\left(P<10^{-8}\right)$, suggesting that GWAS signals may encompass the collective quantitative contributions of large numbers of regulatory variants. Of high priority is determining which specific variants within regulatory DNA functionally impact DNA:protein interactions, local chromatin architecture, and the regulation of target genes (Maurano et al. 2012a). Going forward, ENCODE is well-positioned to contribute substantially to this effort. However, to achieve the highest utility for analysis of disease studies, three challenges confront ENCODE. First, care and coordination must be exercised in selecting cell and tissue types, ideally with close input from the disease communities. Second, selection of transcription factors should be well-matched to known aspects of disease physiology or that of pathogenic cell types. Finally, continuously updated maps of connections between distal regulatory DNA and its target gene(s) must be made available in a format that facilitates integration with GWAS variants.

Another disease area in which ENCODE is poised to yield important insights is cancer. Approximately 40 cancer lines of varying origin have been studied using one or more ENCODE methodologies. Three findings stand out: first, that cancer lines harbor a large number of regulatory DNA regions that are not seen in normal cells (Song et al. 2011; Akhtar-Zaidi et al. 2012; Thurman et al. 2012; Vernot et al. 2012). Second, somatic variation in regulatory DNA of cancer cell lines is unequally distributed, with certain neoplasms having significantly higher rates of somatic regulatory variation (The ENCODE Project Consortium 2012). Additionally, the regulatory DNA of immortal malignant cells (and ES cells) harbors increased germline mutation rates (Thurman et al. 2012; Vernot et al. 2012). How or whether these two processes are connected is unknown. Third, the occupancy landscape of CTCF-and possibly other TFs-differs substantially between normal and immortal cells, a proportion of which is linked to DNA methylation patterns ( $\mathrm{H}$ Wang et al. 2012). In general, many key observations have been enabled by the concurrent availability of cancer genome sequencing data from The Cancer Genome Atlas (TCGA). To develop these observations further, it would seem logical for ENCODE to align future cancer cell type selections as closely as possible with TCGA. 


\section{Trends shaping ENCODE data and their interpretation}

\section{From regions to bases}

At a mechanistic level, most genomic processes operate with nucleotide precision. Currently, however, most ENCODE annotations define regions of tens to hundreds of bases. Closing this resolution gap will be a major challenge going forward. Sites of RNA transcription initiation and termination can generally be mapped at nucleotide level using current approaches. Mapping of DNase I hypersensitivity peaks is giving way to genomic DNase I footprinting (Neph et al. 2012b), which provides nucleotide resolution mapping of protein occupancy sites. However, ChIP-based approaches still require inference. For example, conventional transcription factor ChIP-seq data can only infer that a peak or region contains the protein of interest, with the zone of inference typically spanning $200-300 \mathrm{bp}$. In cases in which a recognition sequence for the cognate factor is extant, it tends to underlie the peak signal. However, roughly half of ENCODE ChIP-seq peaks lack a cognate motif, and in the case of certain individual factors, the proportion of motif-less peaks may exceed 90\% (J Wang et al. 2012b). Most of these cases are likely due to indirect occupancy through protein-protein versus protein-DNA interactions. The recently described ChIP-exo approach (Rhee and Pugh 2011) has the potential to increase substantially the resolution with which binding sites can be localized by ChIP-seq. However, its sample requirements are high, and it is unclear whether it can be applied to most TFs with the same success as seen with the high-occupancy factor CTCF, for which a very high proportion of the occupancy sites harbor clear recognition sequences. ChIP-exo also does not address the direct versus indirect occupancy dilemma.

The case of histone modifications and variants is more complicated. Because modifications or variants are typically distributed across multiple sequential nucleosomes, resolution to nucleosome level is probably sufficient for most needs. This should be straightforward for focally distributed modifications that typically span a small number of nucleosomes (e.g., H3K4me3, H3K27ac, $\mathrm{H} 3 \mathrm{~K} 9 \mathrm{ac}$ ) and lie immediately adjacent to regulatory DNA regions such as promoters and enhancers. Nucleosomes are well-positioned in these regions (Fu et al. 2008), providing a good substrate for single-nucleosome resolution. In more distal regions, where positioning breaks down, it may not be possible to achieve this resolution, nor is it necessarily required because many of the marks found away from active regulatory DNA are widely distributed.

One solution to the resolution dilemma for both TF and histone modification ChIP-seq is coupling them with chromatin accessibility. For example, coupling TF ChIP-seq and genomic footprinting data from the same cell type enables discrimination of direct versus indirect occupancy sites (Neph et al. 2012b). It should also be possible to couple chromatin accessibility with histone modification data to increase the effective resolution of the latter, at least in the vicinity of regulatory DNA.

\section{Man versus machine}

ENCODE data are a natural substrate for pattern discovery via machine learning. In 2007, the application of machine learning techniques to ENCODE Pilot Project data was still nascent, with many approaches such as hidden Markov models adapted from gene-finding applications, or coupled with basic segmentation approaches to integrate across different data types (Thurman et al.
2007). In contrast, nearly every section of the ENCODE integrative paper resulting from the production phase data was driven by machine learning approaches, ranging from advanced segmentation algorithms capable of handling large numbers of diverse data types simultaneously (Ernst and Kellis 2012; Hoffman et al. 2012), to self-organizing maps (A Mortazavi, S Pepke, G Marinov, and B Wold, in prep.), to other hybrid or specialized approaches (The ENCODE Project Consortium 2012). A fundamental result from these approaches was essentially an ab initio demonstration that discrete classes of functional elements are, in fact, encoded by the genome in a manner that matches our long-held perceptions, and that they merely need the right combination of assays to expose them. Different approaches to genomic segmentation essentially converged on the same conclusions concerning specific classes of genomic features including promoters, exons, $3^{\prime}$ ends of genes, CTCF-occupied sites, and even some classes of enhancers. A tacit assumption has been that as more data sets become available, both the power and the resolution of machine learning approaches will increase, somewhat akin to adding more species in a comparative genomic analysis.

A key point remains, however, that the recognition of biological meaning in the output states of machine learning applications is still almost entirely dependent on human-driven syntheses. It is perhaps instructive to observe that the field of gene annotation - the birthplace of genome-directed machine learninghas come to favor the manual curation-driven approach embodied in GENCODE, in which automated algorithms play largely a supporting role. An open question is whether, or to what degree, the assignment of biological meaning to machine-learned states can itself be automated through systematic incorporation of the vast electronic literature.

Whereas ENCODE's current efforts are focused on the integration of biochemical features, the sheer volume of data now available may enable a renaissance in sequence-driven annotation. It is currently unknown to what degree ENCODE-enabled annotations can be derived directly from the primary genome sequence itself (Noble et al. 2005). In parallel with ENCODE, a number of efforts have focused on de novo annotation of enhancers or transcription factor-bound regions by combining conservation, transcription factor recognition motifs, and gene expression (Pennacchio et al. 2007; Busser et al. 2012). The extensive availability of ENCODE-type data for both human and mouse (The Mouse ENCODE Consortium 2012) now provides rich training sets to enable a new generation of machine learning applications (Arvey et al. 2012). Particularly promising is the ability not only to discern complex features such as enhancers directly from sequence data, but also to discriminate those active in different cellular environments (Lee et al. 2011). Aside from understanding the complex and subtle combinations of sequence features underlying ENCODE annotations, such approaches may extend ENCODE to regions of the genome that specify functional elements active in cell populations that are not feasible-either operationally or economically_to address experimentally.

\section{Seeing the big picture}

For most genomic data, the interpretation of biological meaning is closely linked with data visualization. Understanding how different signals are distributed relative to well-studied genes, transcripts, and regulatory DNA regions provides compelling insights into the meaning of different data types, both alone and in combination. In 2007, most ENCODE data tracks could be listed com- 
fortably within the center section of a small poster (The ENCODE Project Consortium 2007). By 2009, it had become apparent that simply calling up the data tracks generated by an individual data production center-let alone the entire consortium-was no longer tenable. Data visualization thus emerged as a major challenge, and yet one that attracted relatively few resources-understandably since major efforts were being directed simply to understand how any single ENCODE data type was to be processed properly in the first place.

Data visualization presents challenges at multiple levels, from logical organization to visual representation. Unfortunately, it is difficult to escape the verdict that ENCODE has fallen short of community expectations at both of these levels. ENCODE data sets themselves are currently difficult to locate, and common tasks aiming to represent large numbers of data tracks-such as visualizing the same data type across a range of cell types, or visualizing many different data types such as TFs within the same cell type-frequently overwhelm the current genome browser paradigm. Compounding the problem, ENCODE data no longer exist in isolation. Other large-scale data generation programs such as the Roadmap Epigenomics Project (Roadmap) (Bernstein et al. 2010), which began running in parallel with ENCODE in late 2008, are producing large volumes of many of the same data types studied by ENCODE, such as DNase I hypersensitivity and histone modifications for different cell and tissue types. More often than not, it is desirable to examine the entire range of a given data type, which requires close integration of ENCODE data with Roadmap and other sources.

Some efforts to redress these issues are under way. To address logical organization and integration with Roadmap data, a combined ENCODE-Roadmap genome browser has been implemented (http://www.epigenomebrowser.org). Beginning in 2010, the Roadmap program invested targeted resources in a new generation of visualization tools designed to facilitate the display and manipulation of large numbers of data tracks. As a result, a variety of novel interfaces are now becoming available such as the epigenome visualization hub (Zhou et al. 2011). In addition, new types of data exploration tools are being developed which will enable pattern-based exploration of ENCODE or Roadmap data sets. In many cases, tools have been tied to the UCSC Genome Browser infrastructure. But it is only a matter of time before ENCODE data become formatted for new "lightweight" genome browsers such as JBrowse (Skinner et al. 2009) that permit smooth scaling from bases to chromosomes, and dynamic reorganization and condensation of large numbers of data tracks. Perhaps the greatest visualization challenge is still imminent: As ENCODE transitions from a one- to a three-dimensional view of genome function, completely new tools and modes of representation will be required. Presently, few appreciate the depth of this problem, and thus little systematic effort is being devoted to visualization apart from first-generation utilities developed by the leading data producers (Lajoie et al. 2009).

\section{Signal and noise}

Virtually all data resulting from high-throughput assays have a component of noise. The introduction of phred quality scores (Ewing et al. 2008) for Sanger sequencing played a key role in the human genome project, since they enabled both the monitoring of data quality within a single production center and the direct comparison of data generated by independent producers. Maximizing the signal-to-noise ratio of the genomic enrichment assays used by ENCODE is of paramount importance in the context of generating reference data that will be widely used by the community. "Clean," high-quality data with high signal-to-noise ratios enable both more accurate delineation of individual elements and increased sensitivity (i.e., the recognition of weaker elements that would otherwise be lost in the noise). High-quality data are particularly vital when deep sequencing can yield additional information such as TF footprints (Neph et al. 2012b).

To date, genomic enrichment assays have lacked quality metrics analogous to the phred score. To address this deficit, both ENCODE and Roadmap have active efforts devoted toward development and application of data quality metrics, as well as the formulation of end-to-end experimental standards for ChIP-seq (Landt et al. 2012) and other data types. It is thus anticipated that emerging quality scores for genomic enrichment assays will have a positive impact on the overall quality of ENCODE data, their utility for the community, and their interoperability with data from diverse laboratories.

\section{The evolution of conservation}

At the outset of ENCODE in 2003, it was widely assumed that evolutionary conservation would prove to be the ultimate arbiter of functional elements in the human genome sequence-all that was lacking was a sufficiently deep sampling of vertebrate genomes for comparative analysis. Correspondingly, highly conserved noncoding sequences were frequently equated with regulatory DNA. For a variety of reasons, both of these expectations missed the mark widely. Following on studies of transcriptional regulation in the RET locus (Fisher et al. 2006), The ENCODE Pilot Project raised a general alarm: Most elements defined by biochemical signatures lacked strong evolutionary conservation (The ENCODE Project Consortium 2007). Conversely, most highly conserved elements escaped annotation using biochemical or other functional assays (Attanasio et al. 2008; McGaughey et al. 2008; Taher et al. 2011). These initial findings have been considerably amplified by the vast volume of data accumulated during the current production phase (The ENCODE Project Consortium 2012) and by other functional studies (Blow et al. 2012). Using conventional measures, most ENCODE-defined elements are poorly conserved, or negligibly so. The number of highly conserved noncoding sequences with an overlapping biochemical function is considerably higher (now roughly half vs. $<10 \%$ [proportionally] after the pilot phase)-although this increase is largely a byproduct of the expanded genomic space annotated by ENCODE, without much enrichment for conserved elements. Complicating this picture, many elements lacking strong conventional signatures of purifying evolutionary selection nonetheless appear to be under constraint in human populations (Vernot et al. 2012).

What conclusions should we draw? On a practical level, the ability to measure function at scale has minimized the role of conservation as a discovery tool. But it has also exposed our ignorance concerning the evolutionary forces shaping the genome, particularly in noncoding regions. The fact that per-nucleotide evolutionary conservation, in combination with nucleotide-level DNA accessibility, can accurately trace a protein-DNA binding interface (Neph et al. 2012b) suggests that the operation of purifying selection is vastly more subtle and complexly structured than had been previously assumed. Moreover, nucleotide-level evolutionary conservation is by itself a poor predictor of functional regulatory variation (Maurano et al. 2012b). However, engrained habits of thought are difficult to escape, and highly conserved 
noncoding elements are still regularly conflated with regulatory elements (Lowe et al. 2011). Clearly, new models of evolutionary conservation are needed to explain the subtleties of regulatory DNA, and the vast trove of ENCODE data provides an unprecedented opportunity for novel and creative syntheses.

A new entry that promises to reshape the conservation conversation is The Mouse ENCODE Project, from which substantial data are already becoming available (Kim et al. 2012; The Mouse ENCODE Consortium 2012; Shen et al. 2012). These data, which have been generated with the same core experimental pipelines used for human ENCODE, will for the first time enable systematic, genome-wide connections between both sequence and the diverse functional modalities encoded within each species' genome.

\section{The road ahead}

ENCODE has made many seminal contributions and is poised for continued success. However, many challenges remain. Prominent among them are two. First is the question of function itself: How will ENCODE-defined elements be assigned a specific functional property (or properties)? Second, how will ENCODE maximize its utility for the broader scientific community?

\section{Localization versus function}

The pre-genome era provided a simple reductionist formula for precisely localizing functional elements and their key internal components: identify, truncate, test-repeat. We learned that operationally defined functional elements such as enhancers and promoters comprise linearly ordered collections of recognition sequences for DNA-binding proteins-the atoms of the regulatory DNA universe. The genomic scale-up of biochemical signature mapping under ENCODE dramatically reshaped this formula, with the ability to delineate likely functional elements greatly outstripping any capacity for defining their functional characteristics through directed experimentation. The result has been a boon for sequence-driven analyses, from regulatory motif derivation to comparative and population genomics.

However, it has also given rise to a broad tendency to think of all elements of a biochemically defined class as having the same functional properties. For example, genomic occupancy by the poly-zinc finger transcriptional regulator CTCF is a prominent feature of experimentally defined enhancer blockers and chromatin boundary elements, as well as bifunctional elements (Gaszner and Felsenfeld 2006). Yet it has now become commonplace to find any CTCF occupancy sites obtained by ChIP-seq referred to as "insulators" without any further specification-and without regard to the well-documented involvement of promoterbound CTCF in transcriptional control (Klenova et al. 1993). Compounding this complexity, ENCODE has now made available data sets encompassing CTCF occupancy across large numbers of cell types (The ENCODE Project Consortium 2012), revealing substantial diversity in occupancy patterns that reflect important differences in regulation and likely in function $(\mathrm{H}$ Wang et al. 2012). Both the sheer number and diversity of these elements argue strongly against ascribing a monolithic functional activity.

A similar situation obtains in the case of enhancers-classically, elements that mediate transcriptional up-regulation, frequently acting at considerable distance from their target gene(s) (Maston et al. 2006). Analysis of ENCODE pilot project data revealed a high ratio of mono- to trimethylated $\mathrm{H} 3 \mathrm{~K} 4$ at a subset of distal DNase I hypersensitive sites (The ENCODE Project Consortium 2007) and at sites of occupancy by the EP300 (also known as p300) acetyltransferase (Heintzman et al. 2007). In spite of the lack of rigorous functional validation, it has now become de rigueur to refer to any region of the genome that exhibits this combination of modifications as an "enhancer" (Heintzman et al. 2009), and further to characterize "strong" and "weak" enhancers merely on the basis of the intensity of the chromatin modification signal (Ernst et al. 2011), or to designate "poised enhancers" (Creyghton et al. 2010) or other subcategorizations based purely on the fine parsing of histone modification patterns (Zentner et al. 2011).

These examples illustrate a natural temptation to equate activity with patterning of epigenomic features. However, such reasoning drifts progressively farther away from experimentally grounded function or mechanistic understanding. The sheer diversity of cross-cell-type regulatory patterning evident in distal regulatory DNA uncovered by ENCODE (Song et al. 2011; Thurman et al. 2012) suggests tremendous heterogeneity and functional diversity. ENCODE is thus in a unique position to promote clearer terminology that separates the identification of functional elements per se from the ascription of specific functional activities using historical experimentally defined categories, and also to dissuade the ascription of very specific functions based on a biochemical signature in place of a deeper mechanistic understanding.

\section{Functional validation: What, how, and how much?}

The lack of extensive classical functional validation performed by ENCODE to date is understandable, given the chasm between the number of biochemically defined elements and the throughput of traditional experimental approaches. But what kinds of elements to validate, how to validate them, and how much of each will be considered definitive? Certainly we cannot expect such an effort to be comprehensive; there are too many elements defined in too many cellular contexts ever to validate individually. A logical approach is class-based validation, with the aim to determine, with statistical rigor, how many members of a given class with given biochemically defined features have a specific functional property, in order that a reliable statement may be made. However, it is presently far from clear that we know how properly to categorize the elements we have. Given their diversity, it is likely that a far larger number will need to be examined than would be feasible with conventional methods. In the case of transcriptional enhancer assay by transient transfection, newer high-throughput approaches are emerging (Melnikov et al. 2012; Patwardhan et al. 2012). However, these impose significant size constraints that restrict their utility. Moreover, the drawbacks of conventional transient assays are well known, most notably the fact that many elements require a chromatin context to function, or a particular primary cellular environment not amenable to transfection.

If significant time and effort is to be invested in highthroughput functional validation, it should be definitive. An emerging alternative that fits this requirement is reverse genetics in an isogenic setting. Once unthinkable for the human genome, knockout of ENCODE-defined regulatory elements is now readily feasible given rapid advances in genome editing technology such as zinc-finger and TAL effector-like nucleases (Doyon et al. 2011; Miller et al. 2011; J Wang et al. 2012a). This technology is currently at a scaling stage (Reyon et al. 2012); given the proper application of resources, thousands of well-designed experiments could reasonably be envisioned over the course of the next phase of ENCODE. Genome editing is well-published in the ENCODE Tier 1 cell type K562 and has the additional advantage of creating a per- 
manent reagent (the knockout line) that can be used for more detailed functional characterization by the community.

\section{Completeness: What does it mean?}

ENCODE was founded with the ultimate objective of amassing a complete catalog of functional elements encoded by the human genome. Nine years on, we are beginning to appreciate the true scope of this lofty goal. More of the human genome sequence appears to be used for some reproducible, biochemically defined activity than was previously imagined. Contrary to the initial expectations of many, the overwhelming majority of these activities appear to be state-specific_-either restricted to specific cell types or lineages, or evokable in response to a stimulus such as interferon. As such, even if we were in possession of technologies with perfect sensitivity in a given cellular context, the sheer diversity of cell types and states is daunting.

It is becoming increasingly clear that functional annotation of the genome entails understanding not only that a particular stretch of DNA encodes a given type of element active in some cell type, but how that encoding is interpreted in different cellular environments. For example, it is widely acknowledged that the same DNA element may be recognized by different (generally related) transcription factors in different cellular environments, with alternative functional consequences. Additionally, we now know that the biochemical signatures of many ENCODE-defined elements exhibit complex trans-cellular patterns of activity (The ENCODE Project Consortium 2012; Thurman et al. 2012), which may be accompanied by functional behaviors such as an enhancer interacting with different target genes (Sanyal et al. 2012; Thurman et al. 2012). Together, these observations suggest that the genome may, in fact, be extensively multiply encoded-i.e., that the same DNA element gives rise to different activities in different cell types. This possibility challenges our current notions of annotation, which are still rooted in a linear world, and cautions against formulating definitions of completeness based on older models such as the delineation of protein-coding genes.

\section{Maximizing utility for the community}

The transition from The ENCODE Pilot Project to the production phase was dominated by technology. Based on many of the trends discussed above, one may predict that the transition from the current production phase to the next will be dominated by utility. Given what we now know about the potential for ENCODE to illuminate not only the genome sequence itself, but also the findings emerging from parallel efforts such as GWAS and TCGA, care must be taken to maximize synergies through careful selection of biological targets and highly coordinated action that maximizes the data generated for each cell or tissue. The high cell requirements entailed by extensive transcription factor ChIP-seq profiling or subcellular RNA fractionation experiments entailed an initial focus on a common set of mainly immortal cells. This has contributed to a perception that ENCODE is largely a cell linecentered endeavor, with limited relevance for many widely studied biological processes. However, overall ENCODE has sampled a vast range of primary cell types-indeed, these outnumber immortalized cell lines nearly 3-to-1 (The ENCODE Project Consortium 2012; Thurman et al. 2012). The potential of ENCODE to contribute to diverse community endeavors is thus now very broad and will be expanded further in the coming production phase as more primary cells enter the experimental pipelines and additional data types such as DNA methylation or maps of RNA-binding proteins become widely available.

ENCODE must recognize and face its awareness problem straight on. ENCODE publications have been cited thousands of times. And yet, broad swathes of the community-even leadingedge laboratories-are unaware of what the project has produced or how to access and interpret the data. A remedy for this situation will not appear spontaneously and will require the intimate involvement of data producers as well as analysts and end users. The only certainty is that if consistent emphasis is not placed on the goal of increasing awareness, and clear milestones defined, little if any progress will be made.

ENCODE is undergoing a transformation from a loosely connected set of annotations to an integrated tool that collectively provides a unique lens through which to view genome function. In this sense, it is gradually transforming from a collection of data into a new kind of tool-almost a type of software that can "operate" on other genomic data types. Indeed, new applications that leverage ENCODE data in this way are already emerging from within the Consortium (Boyle et al. 2012; Ward and Kellis 2012), and one anticipates that many others from diverse community sources are either on the way or will be stimulated as a result of the current suite of ENCODE publications.

\section{Acknowledgments}

I thank the anonymous reviewers and the Editor for helpful suggestions; Sam John for insightful discussions and critical reading of the manuscript; and Richard Humbert for help with references. This work was supported in part by NIH grant U54HG004592.

\section{References}

Affymetrix/Cold Spring Harbor Laboratory ENCODE Transcriptome Project. 2009. Post-transcriptional processing generates a diversity of 5'-modified long and short RNAs. Nature 457: 1028-1032.

Akhtar-Zaidi B, Cowper-Sal-lari R, Corradin O, Saiakhova A, Bartels CF, Balasubramanian D, Myeroff L, Lutterbaugh J, Jarrar A, Kalady MF, et al. 2012. Epigenomic enhancer profiling defines a signature of colon cancer. Science 336: 736-739.

Arvey A, Agius P, Noble WS, Leslie C. 2012. Sequence and chromatin determinants of cell-type-specific transcription factor binding. Genome Res (this issue). doi: 10.1101/gr.127712.111.

Attanasio C, Reymond A, Humbert R, Lyle R, Kuehn MS, Neph S, Sabo PJ, Goldy J, Weaver M, Haydock A, et al. 2008. Assaying the regulatory potential of mammalian conserved non-coding sequences in human cells. Genome Biol 9: R168. doi: 10.1186/gb-2008-9-12-r168.

Banerji J, Olson L, Schaffner W. 1983. A lymphocyte-specific cellular enhancer is located downstream of the joining region in immunoglobulin heavy chain genes. Cell 33: 729-740.

Barski A, Cuddapah S, Cui K, Roh TY, Schones DE, Wang Z, Wei G, Chepelev I, Zhao K. 2007. High-resolution profiling of histone methylations in the human genome. Cell 129: 823-837.

Bernstein BE, Humphrey EL, Erlich RL, Schneider R, Bouman P, Liu JS, Kouzarides T, Schreiber SL. 2002. Methylation of histone H3 Lys 4 in coding regions of active genes. Proc Natl Acad Sci 99: 8695-8700.

Bernstein BE, Kamal M, Lindblad-Toh K, Bekiranov S, Bailey DK, Huebert DJ, McMahon S, Karlsson EK, Kulbokas EJ III, Gingeras TR, et al. 2005. Genomic maps and comparative analysis of histone modifications in human and mouse. Cell 120: 169-181.

Bernstein BE, Stamatoyannopoulos JA, Costello JF, Ren B, Milosavljevic A, Meissner A, Kellis M, Marra MA, Beaudet AL, Ecker JR, et al. 2010. The NIH Roadmap Epigenomics Mapping Consortium. Nat Biotechnol 28: 1045-1048.

Blow MJ, McCulley DJ, Li Z, Zhang T, Akiyama JA, Holt A, Plajzer-Frick I, Shoukry M, Wright C, Chen F, et al. 2012. ChIP-Seq identification of weakly conserved heart enhancers. Nat Genet 42: 806-810.

Boyle AP, Hong EL, Hariharan M, Cheng Y, Schaub MA, Kasowski M, Karczewski KJ, Park J, Hitz BC, Weng S, et al. 2012. Annotation of 
functional variation in personal genomes using RegulomeDB. Genome Res (this issue). doi: 10.1101/gr.137323.112.

Busser BW, Taher L, Kim Y, Tansey T, Bloom MJ, Ovcharenko I, Michelson AM. 2012. A machine learning approach for identifying novel cell typespecific transcriptional regulators of myogenesis. PLoS Genet 8: e1002531. doi: 10.1371/journal.pgen.1002531.

ChengJ, Kapranov P, Drenkow J, Dike S, Brubaker S, Patel S, Long J, Stern D, Tammana H, Helt G, et al. 2005. Transcriptional maps of 10 human chromosomes at 5-nucleotide resolution. Science 308: 1149-1154.

Chung JH, Whiteley M, Felsenfeld G. 1993. A 5' element of the chicken $\beta$-globin domain serves as an insulator in human erythroid cells and protects against position effect in Drosophila. Cell 74: 505-514.

Clark MB, Amaral PP, Schlesinger FJ, Dinger ME, Taft RJ, Rinn JL, Ponting CP, Stadler PF, Morris KV, Morillon A, et al. 2011. The reality of pervasive transcription. PLoS Biol 9: e1000625; discussion e1001102. doi: 10.1371/journal.pbio.1000625.

Creyghton MP, Cheng AW, Welstead GG, Kooistra T, Carey BW, Steine EJ, Hanna J, Lodato MA, Frampton GM, Sharp PA, et al. 2010. Histone H3K27ac separates active from poised enhancers and predicts developmental state. Proc Natl Acad Sci 107: 21931-21936.

de Wit E, de Laat W. 2012. A decade of 3C technologies: Insights into nuclear organization. Genes Dev 26: 11-24.

Dekker J, Rippe K, Dekker M, Kleckner N. 2002. Capturing chromosome conformation. Science 295: 1306-1311.

Derrien T, Johnson R, Bussotti G, Tanzer A, Djebali S, Tilgner H, Guernec G, Martin D, Merkel A, Knowles DG, et al. 2012. The GENCODE v7 catalog of human long noncoding RNAs: Analysis of their gene structure, evolution, and expression. Genome Res (this issue). doi: 10.1101/ gr.132159.111.

Dixon JR, Selvaraj S, Yue F, Kim A, Li Y, Shen Y, Hu M, Liu JS, Ren B. 2012. Topological domains in mammalian genomes identified by analysis of chromatin interactions. Nature 485: 376-380.

Djebali S, Davis CA, Merkel A, Dobin A, Lassmann T, Mortazavi AM, Tanzer A, Lagarde J, Lin W, Schlesinger F, et al. 2012a. Landscape of transcription in human cells. Nature (in press).

Djebali S, Lagarde J, Kapranov P, Lacroix V, Borel C, Mudge JM, Howald C, Foissac S, Ucla C, Chrast J, et al. 2012b. Evidence for transcript network composed of chimeric RNAs in human cells. PLOS ONE 7: e28213. doi: 10.1371/journal.pone.0028213.

Dostie J, Richmond TA, Arnaout RA, Selzer RR, Lee WL, Honan TA, Rubio ED, Krumm A, Lamb J, Nusbaum C, et al. 2006. Chromosome Conformation Capture Carbon Copy (5C): A massively parallel solution for mapping interactions between genomic elements. Genome Res 16: 1299-1309.

Doyon Y, Vo TD, Mendel MC, Greenberg SG, Wang J, Xia DF, Miller JC, Urnov FD, Gregory PD, Holmes MC. 2011. Enhancing zinc-fingernuclease activity with improved obligate heterodimeric architectures. Nat Methods 8: 74-79.

Efroni S, Duttagupta R, Cheng J, Dehghani H, Hoeppner DJ, Dash C, BazettJones DP, Le Grice S, McKay RD, Buetow KH, et al. 2008. Global transcription in pluripotent embryonic stem cells. Cell Stem Cell 2: 437-447.

Emerson BM, Lewis CD, Felsenfeld G. 1985. Interaction of specific nuclear factors with the nuclease-hypersensitive region of the chicken adult $\beta$-globin gene: Nature of the binding domain. Cell 41: 21-30.

The ENCODE Project Consortium. 2007. Identification and analysis of functional elements in 1\% of the human genome by the ENCODE pilot project. Nature 447: 799-816.

The ENCODE Project Consortium. 2012. An integrated encyclopedia of DNA elements in the human genome. Nature (in press).

Ernst J, Kellis M. 2012. ChromHMM: Automating chromatin-state discovery and characterization. Nat Methods 9: 215-216.

Ernst J, Kheradpour P, Mikkelsen TS, Shoresh N, Ward LD, Epstein CB, Zhang X, Wang L, Issner R, Coyne M, et al. 2011. Mapping and analysis of chromatin state dynamics in nine human cell types. Nature 473: $43-49$.

Ewing B, Hillier L, Wendl MC, Green P. 1998. Base-calling of automated sequencer traces using phred. I. Accuracy assessment. Genome Res 8: $175-185$.

Fisher S, Grice EA, Vinton RM, Bessling SL, McCallion AS. 2006. Conservation of RET regulatory function from human to zebrafish without sequence similarity. Science 312: 276-279.

Forrester WC, Thompson C, Elder JT, Groudine M. 1986. A developmentally stable chromatin structure in the human $\beta$-globin gene cluster. Proc Natl Acad Sci 83: 1359-1363.

Frenkel-Morgenstern M, Lacroix V, Ezkurdia I, Levin Y, Gabashvili A, Prilusky J, Del Pozo A, Tress M, Johnson R, Guigo R, et al. 2012. Chimeras taking shape: Potential functions of proteins encoded by chimeric RNA transcripts. Genome Res 22: 1231-1242.

Fu Y, Sinha M, Peterson CL, Weng Z. 2008. The insulator binding protein CTCF positions 20 nucleosomes around its binding sites across the human genome. PLoS Genet 4: e1000138. doi: 10.1371/journal. pgen.1000138.

Gaszner M, Felsenfeld G. 2006. Insulators: Exploiting transcriptional and epigenetic mechanisms. Nat Rev Genet 7: 703-713.

Gaulton KJ, Nammo T, Pasquali L, Simon JM, Giresi PG, Fogarty MP, Panhuis TM, Mieczkowski P, Secchi A, Bosco D, et al. 2010. A map of open chromatin in human pancreatic islets. Nat Genet 42: 255-259.

Gerstein MB, Kundaje A, Hariharan M, Landt SG, Yan K-K, Cheng C, Mu XJ, Khurana E, Rozowsky J, Alexander R, et al. 2012. Architecture of the human regulatory network derived from ENCODE data. Nature (in press)

Gilmour DS, Lis JT. 1984. Detecting protein-DNA interactions in vivo: Distribution of RNA polymerase on specific bacterial genes. Proc Natl Acad Sci 81: 4275-4279.

Grosveld F, van Assendelft GB, Greaves DR, Kollias G. 1987. Positionindependent, high-level expression of the human $\beta$-globin gene in transgenic mice. Cell 51: 975-985.

Harrow J, Frankish A, Gonzalez JM, Tapanari E, Diekhans M, Kokocinski F, Aken BL, Barrell D, Zadissa A, Searle S, et al. 2012. GENCODE: The reference human genome annotation for The ENCODE Project. Genome Res (this issue). doi: 10.1101/gr.135350.111.

Heintzman ND, Stuart RK, Hon G, Fu Y, Ching CW, Hawkins RD, Barrera LO, Van Calcar S, Qu C, Ching KA, et al. 2007. Distinct and predictive chromatin signatures of transcriptional promoters and enhancers in the human genome. Nat Genet 39: 311-318.

Heintzman ND, Hon GC, Hawkins RD, Kheradpour P, Stark A, Harp LF, Ye Z, Lee LK, Stuart RK, Ching CW, et al. 2009. Histone modifications at human enhancers reflect global cell-type-specific gene expression. Nature 459: 108-112.

Hoffman MM, Buske OJ, Wang J, Weng Z, Bilmes JA, Noble WS. 2012. Unsupervised pattern discovery in human chromatin structure through genomic segmentation. Nat Methods 9: 473-476.

Howald C, Tanzer A, Chrast J, Kokocinski F, Derrien T, Walters N, Gonzalez JM, Frankish A, Aken BL, Hourlier T, et al. 2012. Combining RT-PCR-seq and RNA-seq to catalog all genic elements encoded in the human genome. Genome Res (this issue). doi: 10.1101/gr.134478.111.

Jacob F, Monod J. 1961. Genetic regulatory mechanisms in the synthesis of proteins. J Mol Biol 3: 318-356.

Johnson DS, Mortazavi A, Myers RM, Wold B. 2007. Genome-wide mapping of in vivo protein-DNA interactions. Science 316: 1497-1502.

Kapranov P, Willingham AT, Gingeras TR. 2007. Genome-wide transcription and the implications for genomic organization. Nat Rev Genet 8: 413-423.

Kim TK, Hemberg M, Gray JM, Costa AM, Bear DM, Wu J, Harmin DA, Laptewicz M, Barbara-Haley K, Kuersten S, et al. 2012. Widespread transcription at neuronal activity-regulated enhancers. Nature 465: $182-187$.

Klenova EM, Nicolas RH, Paterson HF, Carne AF, Heath CM, Goodwin GH, Neiman PE, Lobanenkov VV. 1993. CTCF, a conserved nuclear factor required for optimal transcriptional activity of the chicken c-myc gene, is an $11-Z n$-finger protein differentially expressed in multiple forms. Mol Cell Biol 13: 7612-7624.

Kolasinska-Zwierz P, Down T, Latorre I, Liu T, Liu XS, Ahringer J. 2009. Differential chromatin marking of introns and expressed exons by H3K36me3. Nat Genet 41: 376-381.

Lajoie BR, van Berkum NL, Sanyal A, Dekker J. 2009. My5C: Web tools for chromosome conformation capture studies. Nat Methods 6: 690-691.

Landt SG, Marinov GK, Kundaje A, Kheradpour P, Pauli F, Batzoglou S, Bernstein BE, Bickel P, Brown JB, Cayting P, et al. 2012. ChIP-seq guidelines and practices of the ENCODE and modENCODE consortia. Genome Res (this issue). doi: 10.1101/gr.136184.111.

Lee DY, Hayes JJ, Pruss D, Wolffe AP. 1993. A positive role for histone acetylation in transcription factor access to nucleosomal DNA. Cell 72: 73-84.

Lee D, Karchin R, Beer MA. 2011. Discriminative prediction of mammalian enhancers from DNA sequence. Genome Res 21: 2167-2180.

Li G, Ruan X, Auerbach RK, Sandhu KS, Zheng M, Wang P, Poh HM, Goh Y, Lim J, Zhang J, et al. 2012. Extensive promoter-centered chromatin interactions provide a topological basis for transcription regulation. Cell 148: $84-98$.

Lieberman-Aiden E, van Berkum NL, Williams L, Imakaev M, Ragoczy T, Telling A, Amit I, Lajoie BR, Sabo PJ, Dorschner MO, et al. 2009. Comprehensive mapping of long-range interactions reveals folding principles of the human genome. Science 326: 289-293.

Lowe CB, Kellis M, Siepel A, Raney BJ, Clamp M, Salama SR, Kingsley DM, Lindblad-Toh K, Haussler D. 2011. Three periods of regulatory innovation during vertebrate evolution. Science 333: 1019-1024

Manak JR, Dike S, Sementchenko V, Kapranov P, Biemar F, Long J, Cheng J, Bell I, Ghosh S, Piccolboni A, et al. 2006. Biological function of unannotated transcription during the early development of Drosophila melanogaster. Nat Genet 38: 1151-1158.

1610 Genome Research

www.genome.org 
Maston GA, Evans SK, Green MR. 2006. Transcriptional regulatory elements in the human genome. Annu Rev Genomics Hum Genet 7: 29-59.

Maurano M, Humbert R, Rynes E, Thurman RE, Haugen E, Wang H, Reynolds AP, Sandstrom R, Qu H, Shafer A, et al. 2012a. Systematic localization of common disease-associated variation in regulatory DNA. Science (in press)

Maurano MT, Wang H, Kutyavin T, Stamatoyannopoulos JA. 2012b. Widespread site-dependent buffering of human regulatory polymorphism. PLoS Genet 8: e1002599. doi: 10.1371/ journal.pgen.1002599.

McGaughey DM, Vinton RM, Huynh J, Al-Saif A, Beer MA, McCallion AS 2008. Metrics of sequence constraint overlook regulatory sequences in an exhaustive analysis at phox 2b. Genome Res 18: 252-260.

Melnikov A, Murugan A, Zhang X, Tesileanu T, Wang L, Rogov P, Feizi S, Gnirke A, Callan CG Jr, Kinney JB, et al. 2012. Systematic dissection and optimization of inducible enhancers in human cells using a massively parallel reporter assay. Nat Biotechnol 30: 271-277.

Mercer TR, Gerhardt DJ, Dinger ME, Crawford J, Trapnell C, Jeddeloh JA, Mattick JS, Rinn JL. 2011. Targeted RNA sequencing reveals the deep complexity of the human transcriptome. Nat Biotechnol 30: 99-104.

Mikkelsen TS, Ku M, Jaffe DB, Issac B, Lieberman E, Giannoukos G, Alvarez P, Brockman W, Kim TK, Koche RP, et al. 2007. Genome-wide maps of chromatin state in pluripotent and lineage-committed cells. Nature 448: 553-560.

Miller JC, Tan S, Qiao G, Barlow KA, Wang J, Xia DF, Meng X, Paschon DE, Leung E, Hinkley SJ, et al. 2011. A TALE nuclease architecture for efficient genome editing. Nat Biotechnol 29: 143-148.

Mortazavi A, Williams BA, McCue K, Schaeffer L, Wold B. 2008. Mapping and quantifying mammalian transcriptomes by RNA-Seq. Nat Methods 5: $621-628$.

The Mouse ENCODE Consortium, Stamatoyannopoulos JA, Snyder M, Hardison R, Ren B, Gingeras T, Gilbert D, Groudine M, Bender M, Kaul R, et al. 2012. An encyclopedia of mouse DNA elements (Mouse ENCODE). Genome Biol (in press).

Neph S, Stergachis AB, Reynolds AP, Sandstrom R, Borenstein E, Stamatoyannopoulos JA. 2012a. Circuitry and dynamics of human transcription factor regulatory networks. Cell (in press).

Neph S, Vierstra J, Stergachis AB, Reynolds AP, Haugen E, Vernot B, Thurman RE, Sandstrom R, Johnson AK, Maurano MT, et al. 2012b. An expansive human regulatory lexicon encoded in transcription factor footprints. Nature (in press)

Noble WS, Kuehn S, Thurman R, Yu M, Stamatoyannopoulos J. 2005 Predicting the in vivo signature of human gene regulatory sequences. Bioinformatics (Suppl 1) 21: i338-i343.

Nora EP, Lajoie BR, Schulz EG, Giorgetti L, Okamoto I, Servant N, Piolot T, van Berkum NL, Meisig J, Sedat J, et al. 2012. Spatial partitioning of the regulatory landscape of the $\mathrm{X}$-inactivation centre. Nature 485: 381-385.

Patwardhan RP, Hiatt JB, Witten DM, Kim MJ, Smith RP, May D, Lee C, Andrie JM, Lee SI, Cooper GM, et al. 2012. Massively parallel functional dissection of mammalian enhancers in vivo. Nat Biotechnol 30: $265-270$.

Pennacchio LA, Loots GG, Nobrega MA, Ovcharenko I. 2007. Predicting tissue-specific enhancers in the human genome. Genome Res 17: 201-211.

Reyon D, Tsai SQ, Khayter C, Foden JA, Sander JD, Joung JK. 2012. FLASH assembly of TALENs for high-throughput genome editing. Nat Biotechnol 30: $460-465$

Rhee HS, Pugh BF. 2011. Comprehensive genome-wide protein-DNA interactions detected at single-nucleotide resolution. Cell 147: 1408-1419.

Robertson G, Hirst M, Bainbridge M, Bilenky M, Zhao Y, Zeng T, Euskirchen G, Bernier B, Varhol R, Delaney A, et al. 2007. Genome-wide profiles of STAT1 DNA association using chromatin immunoprecipitation and massively parallel sequencing. Nat Methods 4: 651-657.

Santos-Rosa H, Schneider R, Bannister AJ, Sherriff J, Bernstein BE, Emre NC, Schreiber SL, Mellor J, Kouzarides T. 2002. Active genes are trimethylated at K4 of histone H3. Nature 419: 407-411.

Sanyal A, Lajoie B, Jain G, Dekker J. 2012. The long-range interaction landscape of gene promoters. Nature (in press).

Schaub MA, Boyle AP, Kundaje A, Batzoglou S, Snyder M. 2012. Linking disease associations with regulatory information in the human genome. Genome Res (this issue). doi: 10.1101/gr.136127.111.

Shen Y, Yue F, McCleary DF, Ye Z, Edsall L, Kuan S, Wagner U, Dixon J, Lee L, Lobanenkov VV, et al. 2012. A map of the cis-regulatory sequences in the mouse genome. Nature doi: 10.1038/nature11243.

Skinner ME, Uzilov AV, Stein LD, Mungall CJ, Holmes IH. 2009. JBrowse: A next-generation genome browser. Genome Res 19: 1630-1638.
Solomon MJ, Varshavsky A. 1985. Formaldehyde-mediated DNA-protein crosslinking: A probe for in vivo chromatin structures. Proc Natl Acad Sci 82: 6470-6474.

Song L, Zhang Z, Grasfeder LL, Boyle AP, Giresi PG, Lee BK, Sheffield NC, Graf S, Huss M, Keefe D, et al. 2011. Open chromatin defined by DNase I and FAIRE identifies regulatory elements that shape cell-type identity. Genome Res 21: 1757-1767.

Stalder J, Larsen A, Engel JD, Dolan M, Groudine M, Weintraub H. 1980. Tissue-specific DNA cleavages in the globin chromatin domain introduced by DNAase I. Cell 20: $451-460$.

Strauss EC, Orkin SH. 1992. In vivo protein-DNA interactions at hypersensitive site 3 of the human $\beta$-globin locus control region. Proc Natl Acad Sci 89: 5809-5813.

Taher L, McGaughey DM, Maragh S, Aneas I, Bessling SL, Miller W, Nobrega MA, McCallion AS, Ovcharenko I. 2011. Genome-wide identification of conserved regulatory function in diverged sequences. Genome Res 21: 1139-1149.

Thurman RE, Day N, Noble WS, Stamatoyannopoulos JA. 2007. Identification of higher-order functional domains in the human ENCODE regions. Genome Res 17: 917-927.

Thurman RE, Rynes E, Humbert R, Vierstra J, Maurano MT, Haugen E, Sheffield NC, Stergachis AB, Wang H, Vernot B, et al. 2012. The accessible chromatin landscape of the human genome. Nature (in press).

Tilgner H, Nikolaou C, Althammer S, Sammeth M, Beato M, Valcarcel J, Guigo R. 2009. Nucleosome positioning as a determinant of exon recognition. Nat Struct Mol Biol 16: 996-1001.

Tilgner H, Knowles DG, Johnson R, Davis CA, Chakrabortty S, Djebali S, Curado J, Snyder M, Gingeras TR, Guigó R. 2012. Deep sequencing of subcellular RNA fractions shows splicing to be predominantly cotranscriptional in the human genome but inefficient for lncRNAs. Genome Res (this issue). doi: 10.1101/gr.134445.111.

Tuan D, Kong S, Hu K. 1992. Transcription of the hypersensitive site HS2 enhancer in erythroid cells. Proc Natl Acad Sci 89: 11219-11223.

Vernot B, Stergachis AB, Maurano MT, Vierstra J, Neph S, Thurman RE, Stamatoyannopoulos JA, Akey JM. 2012. Personal and population genomics of human regulatory variation. Genome Res (this issue). doi: 10.1101/gr.134890.111.

Wang Z, Zang C, Rosenfeld JA, Schones DE, Barski A, Cuddapah S, Cui K, Roh TY, Peng W, Zhang MQ, et al. 2008. Combinatorial patterns of histone acetylations and methylations in the human genome. Nat Genet 40: 897-903.

Wang H, Maurano MT, Qu H, Varley KE, Gertz J, Pauli F, Lee K, Canfield T, Weaver M, Sandstrom R, et al. 2012. Widespread plasticity in CTCF occupancy linked to DNA methylation. Genome Res (this issue). doi: 10.1101/gr.136101.111.

Wang J, Friedman G, Doyon Y, Wang NS, Li CJ, Miller JC, Hua KL, Yan JJ, Babiarz JE, Gregory PD, et al. 2012a. Targeted gene addition to a predetermined site in the human genome using a ZFN-based nicking enzyme. Genome Res 22: 1316-1326.

Wang J, Zhuang J, Iyer S, Lin XY, Whitfield TW, Greven MC, Pierce BG, Dong $\mathrm{X}$, Kundaje A, Cheng Y, et al. 2012b. Sequence features and chromatin structure around the genomic regions bound by 119 human transcription factors. Genome Res (this issue). doi: 10.1101/ gr.139105.112.

Ward LD, Kellis M. 2012. HaploReg: A resource for exploring chromatin states, conservation, and regulatory motif alterations within sets of genetically linked variants. Nucleic Acids Res 40: D930-D934.

Washietl S, Pedersen JS, Korbel JO, Stocsits C, Gruber AR, Hackermuller J, Hertel J, Lindemeyer M, Reiche K, Tanzer A, et al. 2007. Structured RNAs in the ENCODE selected regions of the human genome. Genome Res 17: 852-864.

Weintraub H, Groudine M. 1976. Chromosomal subunits in active genes have an altered conformation. Science 193: 848-856.

Wu C. 1980. The $5^{\prime}$ ends of Drosophila heat shock genes in chromatin are hypersensitive to DNase I. Nature 286: 854-860.

Wu C, Wong YC, Elgin SC. 1979. The chromatin structure of specific genes: II. Disruption of chromatin structure during gene activity. Cell 16: 807814.

Zentner GE, Tesar PJ, Scacheri PC. 2011. Epigenetic signatures distinguish multiple classes of enhancers with distinct cellular functions. Genome Res 21: 1273-1283.

Zhou X, Maricque B, Xie M, Li D, Sundaram V, Martin EA, Koebbe BC, Nielsen C, Hirst M, Farnham P, et al. 2011. The Human Epigenome Browser at Washington University. Nat Methods 8: 989-990. 


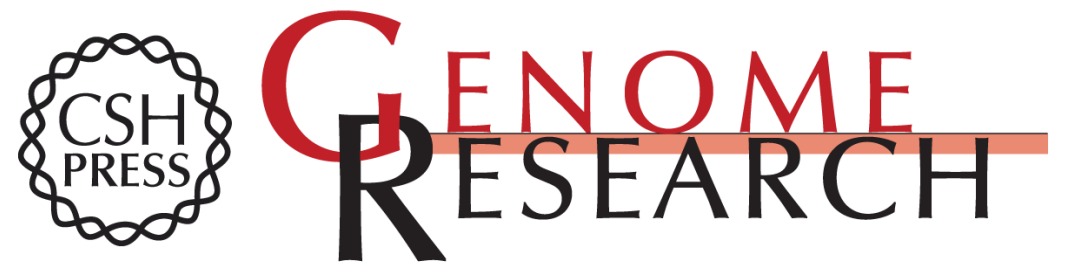

\section{What does our genome encode?}

John A. Stamatoyannopoulos

Genome Res. 2012 22: 1602-1611

Access the most recent version at doi:10.1101/gr.146506.112

\section{Affordable, Accurate} Sequencing.

To subscribe to Genome Research go to: https://genome.cshlp.org/subscriptions 
References This article cites 84 articles, 30 of which can be accessed free at: http://genome.cshlp.org/content/22/9/1602.full.html\#ref-list-1

Articles cited in:

http://genome.cshlp.org/content/22/9/1602.full.html\#related-urls

Open Access Freely available online through the Genome Research Open Access option.

Creative This article is distributed exclusively by Cold Spring Harbor Laboratory Press for the Commons License first six months after the full-issue publication date (see http://genome.cshlp.org/site/misc/terms.xhtml). After six months, it is available under a Creative Commons License (Attribution-NonCommercial 3.0 Unported License), as described at http://creativecommons.org/licenses/by-nc/3.0/.

Email Alerting

Receive free email alerts when new articles cite this article - sign up in the box at the Service top right corner of the article or click here.

\section{Affordable, Accurate Sequencing.}

To subscribe to Genome Research go to: https://genome.cshlp.org/subscriptions 
Related Content Decoding the human genome

Kelly A. Frazer

Genome Res. September, 2012 22: 1599-1601 GENCODE: The reference human genome annotation for The ENCODE Project

Jennifer Harrow, Adam Frankish, Jose M. Gonzalez, et al.

Genome Res. September , 2012 22: 1760-1774 Combining RT-PCR-seq and

RNA-seq to catalog all genic elements encoded in the human genome

Cédric Howald, Andrea Tanzer, Jacqueline Chrast, et al.

Genome Res. September, 2012 22: 1698-1710 The GENCODE v7 catalog of

human long noncoding RNAs: Analysis of their gene structure, evolution, and expression

Thomas Derrien, Rory Johnson, Giovanni Bussotti, et al.

Genome Res. September , 2012 22: 1775-1789 Annotation of functional variation in personal genomes using RegulomeDB

Alan P. Boyle, Eurie L. Hong, Manoj Hariharan, et al.

Genome Res. September , 2012 22: 1790-1797 Toward mapping the biology of

the genome

Stephen Chanock

Genome Res. September , 2012 22: 1612-1615 Sequence features and chromatin structure around the genomic regions bound by 119 human transcription factors

Jie Wang, Jiali Zhuang, Sowmya lyer, et al.

Genome Res. September, 2012 22: 1798-1812 Understanding transcriptional

regulation by integrative analysis of transcription factor binding data

Chao Cheng, Roger Alexander, Renqiang Min, et al.

Genome Res. September , 2012 22: 1658-1667 A highly integrated and complex

PPARGC1A transcription factor binding network in HepG2 cells

Alexandra E. Charos, Brian D. Reed, Debasish Raha, et al.

Genome Res. September , 2012 22: 1668-1679 Widespread plasticity in CTCF

occupancy linked to DNA methylation

Hao Wang, Matthew T. Maurano, Hongzhu Qu, et al.

Genome Res. September, 2012 22: 1680-1688 Predicting cell-typespecific gene

expression from regions of open chromatin

Anirudh Natarajan, Galip Gürkan Yardimci, Nathan C. Sheffield, et al.

Genome Res. September, 2012 22: 1711-1722 Sequence and chromatin

determinants of cell-typespecific transcription factor binding

Aaron Arvey, Phaedra Agius, William Stafford Noble, et al.

Genome Res. September , 2012 22: 1723-1734 Ubiquitous heterogeneity and

asymmetry of the chromatin environment at regulatory elements

Anshul Kundaje, Sofia Kyriazopoulou-Panagiotopoulou, Max Libbrecht, et al.

Genome Res. September, 2012 22: 1735-1747 ChIP-seq guidelines and practices of the ENCODE and modENCODE consortia

Stephen G. Landt, Georgi K. Marinov, Anshul Kundaje, et al.

Genome Res. September , 2012 22: 1813-1831 Personal and population

genomics of human regulatory variation

Benjamin Vernot, Andrew B. Stergachis, Matthew T. Maurano, et al.

Genome Res. September , 2012 22: 1689-1697 Linking disease associations with regulatory information in the human genome

Marc A. Schaub, Alan P. Boyle, Anshul Kundaje, et al.

Genome Res. September , 2012 22: 1748-1759

\section{Affordable, Accurate} Sequencing.

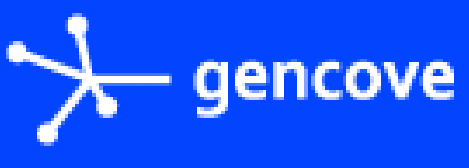

To subscribe to Genome Research go to:

https://genome.cshlp.org/subscriptions 